\title{
Superior Efficacy with Ivermectin 1\% Cream Compared to Metronidazole $0.75 \%$ Cream Contributes to a Better Quality of Life in Patients with Severe Papulopustular Rosacea: A Subanalysis of the Randomized, Investigator-Blinded ATTRACT Study
}

Martin Schaller · Thomas Dirschka $\cdot$ Lajos Kemény · Philippe Briantais ·

Jean Jacovella

Received: June 14, 2016 / Published online: July 18, 2016

(c) The Author(s) 2016. This article is published with open access at Springerlink.com

\section{ABSTRACT}

Introduction: Ivermectin 1\% cream (IVM 1\%) is indicated for the treatment of inflammatory lesions of rosacea. The objective of this subanalysis was to compare IVM $1 \%$ vs. metronidazole $0.75 \%$ cream (MTZ 0.75\%) in the treatment of severe inflammatory lesions of rosacea.

Methods: A subanalysis of the investigator-assessed severe subjects from a Phase 3, investigator-blinded, randomized

On behalf of the Ivermectin Phase III study group.

Enhanced content To view enhanced content for this article go to http://www.medengine.com/Redeem/ A1E4F0604AFDF282.

M. Schaller $(\square)$

Department of Dermatology, Tübingen University

Hospital, Tübingen, Germany

e-mail: martin.schaller@med.uni-tuebingen.de

T. Dirschka

CentroDerm-Clinic, Wuppertal, Germany and

Faculty of Health, University of Witten-Herdecke,

Witten, Germany

L. Kemény

Department of Dermatology and Allergology,

Faculty of Medicine, University of Szeged, Szeged, Hungary

P. Briantais · J. Jacovella

Galderma R\&D, Sophia Antipolis, France study comparing IVM $1 \%$ once daily (QD) with MTZ $0.75 \%$ twice daily (BID) over 16 weeks followed by a 36-week extension period was performed. Efficacy assessments were Dermatology Life Quality Index (DLQI) and EuroQol-5 Dimension (EQ-5D) questionnaires, investigator's global assessment (IGA), subject assessment of rosacea improvement, and inflammatory lesion counts. Adverse events (AEs) were monitored throughout the study.

Results: A total of 161 subjects $(16.7 \%$ of overall study population; 80 IVM $1 \%$ and 81 MTZ $0.75 \%$ ) had an IGA score of 4 at baseline representing severe papulopustular rosacea. Significantly more IVM $1 \%$ subjects had a minimal clinically important difference (MCID, defined as a decrease from baseline of $\geq 4$ points) in DLQI score than MTZ $0.75 \%$ subjects at week $16 \quad(65.4 \%$ vs. $39.2 \%$; $P=0.001)$ and week $52(68.8 \%$ vs. $40.4 \%$; $P=0.003)$. At week 16 , the mean EQ-5D score for the IVM 1\% subjects was higher (better quality of life) than for MTZ $0.75 \%$ subjects (0.941 vs. 0.896). Significantly more IVM $1 \%$ subjects were IGA "clear" or "almost clear" at week 16 compared to MTZ $0.75 \%$ (82.5\% vs. 
63.0\%; $P=0.005)$. Incidence of AEs was comparable between groups.

Conclusion: Better efficacy with IVM 1\% cream (QD) compared to MTZ $0.75 \%$ cream (BID) contributes to an improved quality of life with significantly more patients achieving an MCID in DLQI score at week 16 and higher mean EQ-5D score. IVM $1 \%$ cream is thus a better alternative than MTZ $0.75 \%$ cream for severe papulopustular rosacea patients.

Trial registration: EUDRACT number: 2011-004791-11.

Funding: Galderma R\&D.

Keywords: DLQI; EQ-5D; Inflammatory lesions; Ivermectin; Metronidazole; Randomized clinical trial; Severe papulopustular rosacea; Subanalysis; Topical

\section{INTRODUCTION}

Rosacea is a chronic inflammatory disease that affects around $10 \%$ of the population in Europe [1]. Clinical characteristics include primary features (transient erythema or flushing, fixed/ non-transient erythema, inflammatory papules/pustules, telangiectasia) and secondary features (burning, plaque, edema) in various combinations [2]. As it is readily visible on the face, rosacea has a considerable impact on a patient's quality of life (QoL) [3].

Until recently, patients with severe inflammatory lesions were often treated with antibiotics. However, a European Commission action plan [4] and World Health Organization global action plan [5] have been implemented in recent years to tackle the ever-increasing problem of antimicrobial resistance, including reducing antibiotic use in non-infectious diseases.

Current topical treatments for inflammatory lesions of rosacea (papulopustular) include metronidazole $\quad 0.75 \%$ and $1 \%$ topical formulations and azelaic acid 15\% gel [6]. Topical ivermectin 1\% cream (IVM 1\%; Soolantra $^{\circledR} 1 \%$ cream, Galderma, Lausanne, Switzerland) was approved across Europe in 2015 based on 2 pivotal Phase 3 studies [7] and 1 supportive Phase 3 study [8], involving a total of over 2300 subjects. In the 16-week active-controlled study in subjects with moderate-to-severe papulopustular inflammatory lesions of rosacea, once daily (QD) IVM 1\% was shown to be superior to twice daily (BID) metronidazole $0.75 \%$ cream (MTZ 0.75\%) in reducing inflammatory lesion counts [8]. IVM 1\% demonstrated early onset of efficacy, improved efficacy and longer remission over an existing reference treatment [8, 9], making it a new therapeutic option that meets the needs of rosacea patients.

The objective of this post hoc subanalysis was to compare IVM 1\% cream (QD) with MTZ $0.75 \%$ cream (BID) in the treatment of subjects with investigator-assessed severe inflammatory lesions of rosacea (papulopustular).

\section{METHODS}

\section{Study Design and Subjects}

This was a post hoc subanalysis of a Phase 3, investigator-blinded, randomized, parallel-group, superiority study that previously compared the efficacy and safety of QD IVM $1 \%$ cream with BID MTZ $0.75 \%$ cream in subjects with moderate-to-severe papulopustular rosacea. The study included a 16-week treatment period (Part A) [8] followed by a 36-week extension period to assess relapse among the successfully treated patients (Part B) [9] for a total study period of 52 weeks. The study design has previously been described in detail [8]. 
Table 1 Investigator's global assessment (IGA) scale

\begin{tabular}{lll}
\hline Grade & Score & Clinical description \\
\hline Clear & 0 & No inflammatory lesions present, no erythema \\
Almost clear & 1 & Very few small papules/pustules, very mild erythema present \\
Mild & 2 & Few small papules/pustules, mild erythema \\
Moderate & 3 & Several small or large papules/pustules, moderate erythema \\
Severe & 4 & Numerous small and/or large papules/pustules, severe erythema \\
\hline
\end{tabular}

This post hoc subgroup analysis was performed on study subjects with severe papulopustular rosacea, i.e., an investigator's global assessment (IGA) score (see Table 1) of 4 at baseline.

\section{Treatment}

As previously described, subjects were randomized in a 1:1 ratio to receive either IVM $1 \%$ (QD) or MTZ $0.75 \%$ (BID) for 16 weeks. Study treatment, IVM $1 \%$ or MTZ $0.75 \%$, was discontinued at the end of the 16 -week treatment period and was restarted in the 36-week extension period in the event of relapse (IGA score of at least 2) with treatment being stopped when the IGA score returned to 0 or 1 (multiple retreatment periods were allowed). Study drugs were to be applied in a thin film on the entire face (right and left cheeks, forehead, chin and nose), avoiding the upper and lower eyelids, lips, eyes, and mouth.

\section{Assessments}

EuroQol-5 Dimension (EQ-5D) [10] and Dermatology Life Quality Index (DLQI) [11] QoL questionnaires were completed at baseline, week 16, week 32, and week 52. Subjects evaluated their rosacea improvement at week 16 and week 52. At each study visit, inflammatory lesions (papules and pustules) were counted on five facial regions (forehead, chin, nose, right cheek, left cheek) and the subject's rosacea was graded according to the IGA scale (Table 1). AEs were monitored throughout the study.

\section{Statistical Methods}

The means of the DLQI total score and EQ-5D index were calculated using the definitions for each questionnaire.

The EQ-5D descriptive system consists of five dimensions (mobility, self-care, usual activities, pain/discomfort and anxiety/depression) with three levels each (no problems, some problems, and extreme problems).The EQ-5D results were converted into a single summary index (where $0=$ dead and $1.0=$ full health) by applying Dutch preference weights [12]. An improvement in the EQ-5D score of $\geq 0.074$ vs. baseline is considered as the minimal clinically important difference (MCID) [13].

The 10 DLQI questions had four possible responses from not at all (0) to very much (3) [11] and the total score was calculated by summing the score of each question; the maximum score is 30 (extremely large effect on patient's life) and minimum is 0 (no effect on patient's life). The 10 questions cover 6 domains and DLQI subscale scores were calculated for symptoms and feelings, daily activities, leisure, work and school, personal relationships, and treatment. The changes from 
baseline were analyzed by analysis of covariance, including baseline DLQI as a covariate. A change in DLQI score of at least 4 points is considered MCID and suggests that there has been a meaningful change in the patient's QoL since the previous DLQI measurement [14].

Efficacy endpoints included success rate, expressed as percentage of subjects with IGA rated as 0 or 1 (analyzed by chi $^{2}$ or Fisher's exact test), IGA and percent change in lesion counts (analyzed using Wilcoxon rank sum test); other variables were descriptively analyzed.

Analysis was performed using SAS version 9.2 (SAS Institute Inc., Cary, NC, USA).

\section{Compliance with Ethics Guidelines}

This article is based on a previously conducted study and does not involve any new studies of human or animal subjects performed by any of the authors. Informed consent was received from the patient for publication of the photographs.

\section{RESULTS}

\section{Subject Disposition}

Of the total of 962 randomized subjects (478 IVM 1\%, 484 MTZ 0.75\%), 161 (16.7\%; 80 IVM $1 \%, 81$ MTZ $0.75 \%$ ) had an IGA score of 4 at baseline representing severe inflammatory lesions of rosacea.

\section{Quality of Life}

In this subgroup of 161 subjects with severe inflammatory lesions of rosacea, the mean DLQI total scores at baseline were 7.8 and 6.6 for the IVM $1 \%$ and MTZ $0.75 \%$ groups, respectively, indicating a moderate impact of severe papulopustular rosacea on QoL.

At week 16, a greater reduction from baseline (i.e., a greater improvement in QoL) in the mean DLQI score was observed for IVM 1\% group subjects than MTZ $0.75 \%$ subjects ( -6.0 vs. -3.8 ; $P=0.014)$. Similar results were observed at week 52 ( -6.0 vs. $-4.8 ; P=0.010$; Fig. 1 ).

After 16 weeks treatment, the proportion of subjects with a DLQI score of 0 or 1 representing no detrimental effect on QoL was $69.2 \%$ for the IVM $1 \%$ group vs. $53.2 \%$ for the MTZ $0.75 \%$ group $(P=0.03)$; at week 52 the difference between the 2 groups was even higher at $81.3 \%$ vs. $61.7 \%$, respectively $(P=0.022)$.

At week 16, significantly more IVM $1 \%$ subjects had an MCID of the DLQI score than MTZ $\quad 0.75 \%$ subjects $\quad(65.4 \%$ vs. $39.2 \%$; $P=0.001)$. A similar statistically significant difference was observed at week 52 (68.8\% vs. $40.4 \%$, respectively; $P=0.003$ ).

The greatest improvement in the mean score between baseline and week 16 was observed for the DLQI subscale of symptoms and feelings with a mean score of -2.2 for the IVM $1 \%$ group and -1.6 for the MTZ $0.75 \%$ group $(P=0.03)$.

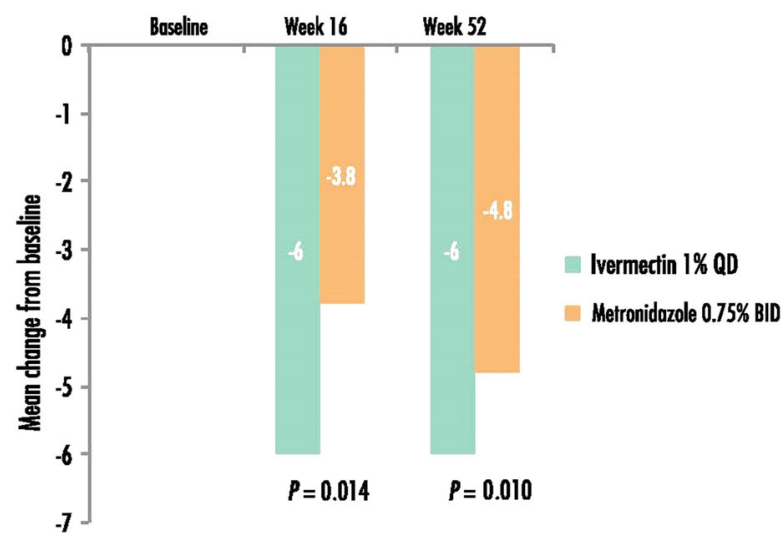

Fig. 1 Mean change from baseline in DLQI score (a lower score indicates better quality of life). The statistical test was adjusted for the baseline values. BID twice daily, $D L Q I$ Dermatology Life Quality Index, $Q D$ once daily 
At week 16, the mean EQ-5D score for the subjects treated with IVM $1 \%$ was higher (indicating a better QoL) than for subjects treated with MTZ $0.75 \%$ (0.941 vs. 0.896) and the mean change from baseline in EQ-5D score was 0.074 vs. $0.069(P=0.032)$ for the IVM $1 \%$ group and the MTZ $0.75 \%$ group, respectively (Fig. 2). Similarly, at week 52, the mean change from baseline was significantly higher for IVM $1 \%$ subjects than MTZ $0.75 \%$ subjects ( 0.103 vs. $0.084 ; P=0.010)$. Subjects with IGA success (grade 0-1) consistently had statistically

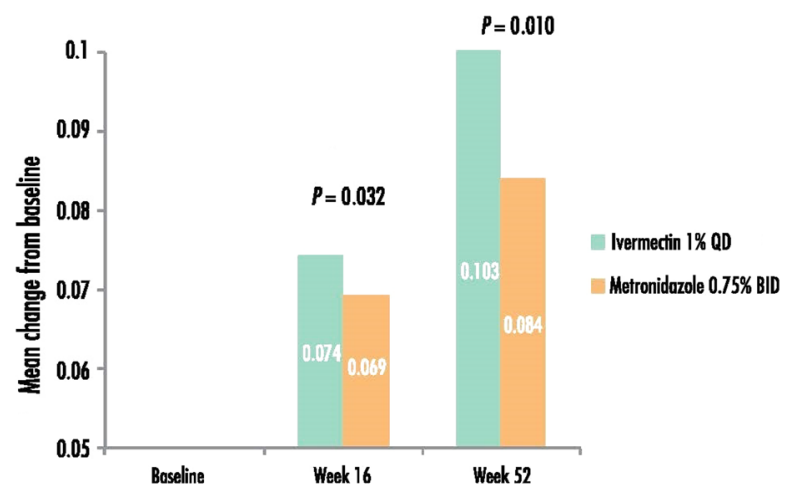

Fig. 2 Mean change from baseline in EQ-5D score (a higher score indicates better quality of life). The statistical test was adjusted for the baseline values. EQ-5D EuroQol-5 Dimension significantly better EQ-5D scores than subjects with IGA failure (grade 2-4; $0.939 \pm 0.131$ vs. $0.870 \pm 0.172 ; P<0.01)$.

The most improved EQ-5D dimensions were pain/discomfort and anxiety/depression. The number of subjects without pain/discomfort was $22.1 \%$ higher in the IVM $1 \%$ group at week 16 compared to baseline, whereas the respective increase was only $5.9 \%$ in the MTZ $0.75 \%$ group.

\section{Efficacy}

For success rate based on an IGA score of 0 or 1 at week 16, more subjects in the IVM 1\% group (66/80 subjects, $82.5 \%$ ) were assessed as success compared to the MTZ $0.75 \%$ group (51/81 subjects, $63.0 \%$ ), i.e., a between-group difference of $19.5 \%$ (Fig. 3).

At week 16, twice as many IVM $1 \%$ subjects (22/80 subjects, 27.5\%) than MTZ $0.75 \%$ subjects (10/81 subjects, $12.3 \%)$ had an IGA score of 0 (clear; Fig. 3).

At week 16, the mean (median) percentage reduction in inflammatory lesion counts from baseline (intent-to-treat last observation carried forward) was higher for the IVM 1\% group at

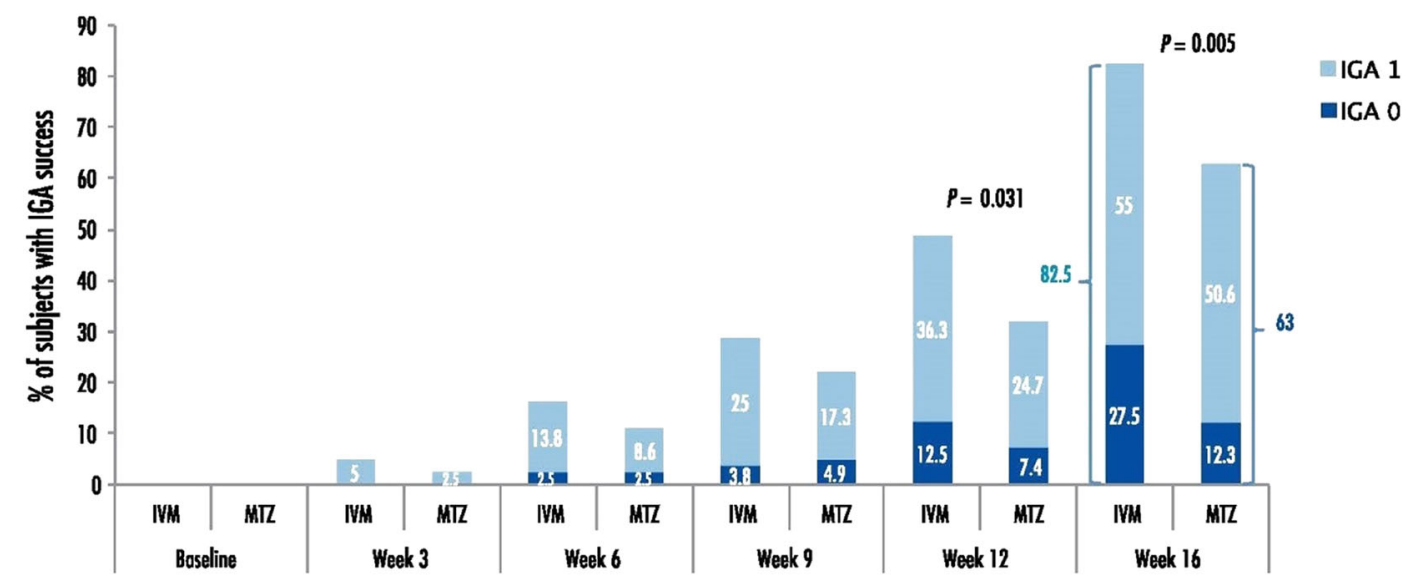

Fig. 3 Success rate based on subjects who achieved an IGA score of 0 (clear) or 1 (almost clear) (intention-to-treat, last observation carried forward). IGA investigator's global

assessment, $I V M$ ivermectin $1 \%$ cream, $M T Z$ metronidazole $0.75 \%$ cream 


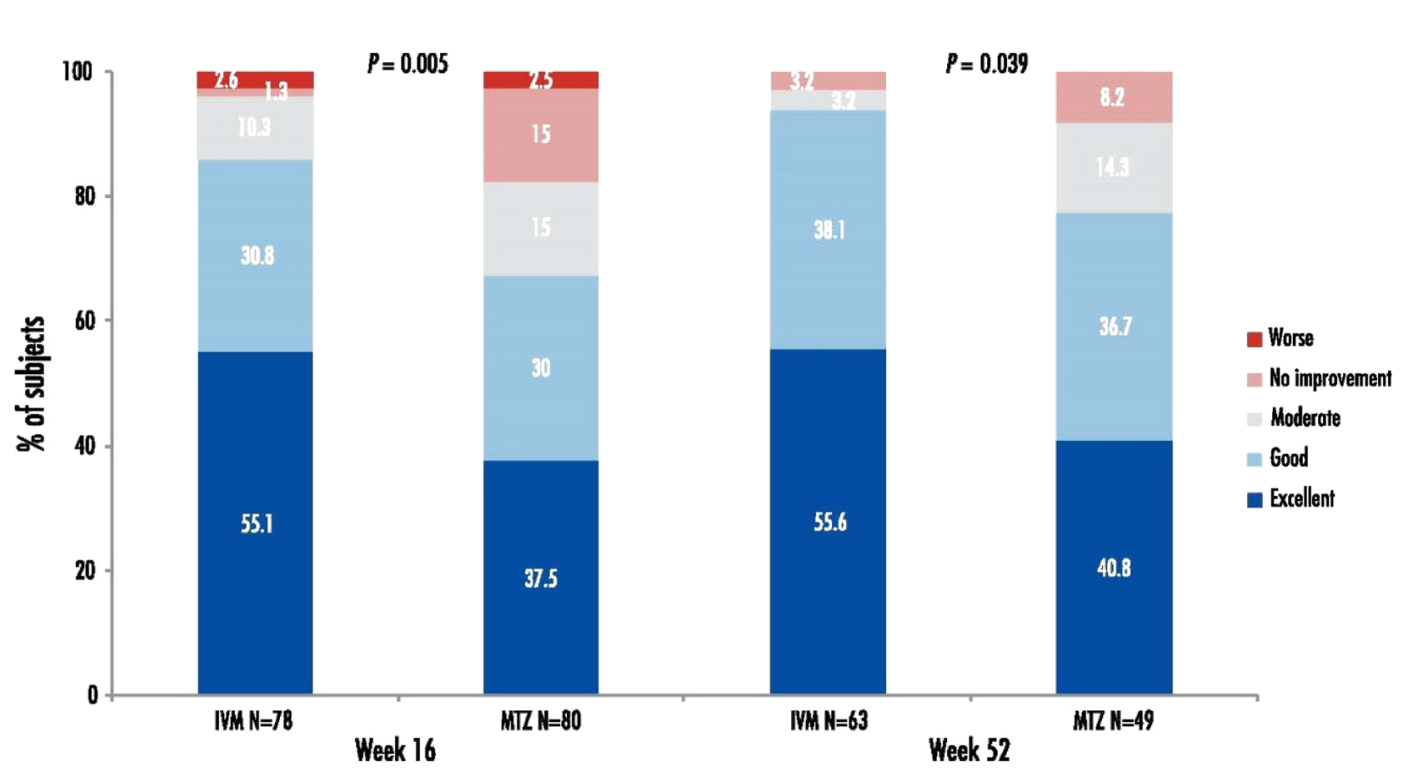

Fig. 4 Subjects' rating of rosacea improvement. $I V M$ ivermectin $1 \%$ cream, $M T Z$ metronidazole $0.75 \%$ cream

$-85.1 \%$ (-93.7\%) compared to the MTZ 0.75\% group at $-75.2 \%(-88.1 \%)(P=0.011)$.

Subjects' rating of rosacea improvement is illustrated in Fig. 4. Patient self-assessment of rosacea improvement at week 16 was good/ excellent for $85.9 \%$ of IVM-treated subjects vs. $67.5 \%$ of MTZ-treated subjects. Similarly, at week $52,93.7 \%$ of subjects in the IVM 1\% group rated their improvement as good/ excellent compared to $77.5 \%$ in the MTZ $0.75 \%$ group.

The overall success rate, i.e., percentage of subjects who were successfully treated over the treatment period of 16 weeks (IGA 0 or 1 ) and never relapsed during the 36-week extension period, was higher in the IVM 1\% group than the MTZ $0.75 \%$ group (23.2\% IVM 1\% vs. $12.3 \%$ MTZ 0.75\%).

\section{Safety}

There were a total of three related adverse events in Part A (rosacea in 1 IVM 1\% subject; allergic dermatitis in 1 MTZ $0.75 \%$ subject; pruritus in 1 MTZ $0.75 \%$ subject) and two related adverse events in Part B (pruritus and rosacea in 1 IVM $1 \%$ subject).

\section{DISCUSSION}

The greater improvement in QoL in the IVM 1\% group than in the MTZ $0.75 \%$ group reflects the superior efficacy with IVM $1 \%$ with a comparable safety profile in this subpopulation of patients with severe inflammatory lesions of rosacea.

Although QoL is inherently subjective in nature, the importance of patient-reported outcomes in evaluating the efficacy and safety of treatments for rosacea was highlighted in the 2015 Cochrane review with "Change in quality of life" being a primary outcome measure [6]. It is important to determine whether changes in physical symptoms interpreted by the clinician translate into measurably significant changes in QoL for the individual. This was the case since mean difference in DLQI scores and EQ-5D scores were consistently statistically significantly better for patients with treatment success than for those with treatment failure. 
Since the extent of improvement in QoL total scores lack a direct clinical meaning, the concept of MCID (defined previously [13, 14]) was used to confirm clinically relevant treatment effectiveness.

More subjects in the IVM 1\% group had an MCID in DLQI score compared with the MTZ $0.75 \%$ group at week $16(65.4 \%$ vs. $39.2 \%$; $P=0.001)$. As all patients who continued into Part B had been treated successfully in Part A whatever the treatment group (i.e., all were IGA 0 or 1 at week 16), it may be expected that the subjects in both groups would be equally satisfied with the treatment in Part B. However, this was not the case since at week 52 , the improvement in proportion of subjects with an MCID in DLQI score (68.8\% IVM 1\% vs. $40.4 \%$ MTZ $0.75 \% ; \quad P=0.003)$ was again significantly greater in the IVM $1 \%$ group, reflecting the clinical superiority of IVM $1 \%$ over the long-term. These results were corroborated with the results obtained using the generic EQ-5D questionnaire, with which the mean change from baseline at week 16 and week 52 were significantly higher in the IVM $1 \%$ group than the MTZ $0.75 \%$ group. The EQ-5D is a very widely used generic questionnaire and it is unusual to observe a statistically significant difference in scores for dermatological diseases, especially with a small sample size.

QoL data collected at week 32 could not be directly compared between the IVM $1 \%$ and MTZ $0.75 \%$ groups as the level of exposure to the two drugs was heterogeneous at this time point. In fact, more subjects in the MTZ 0.75\% group had already been re-treated at week 32 compared to the IVM $1 \%$ group due to the longer remission time for the IVM $1 \%$ group, as reported previously [9].

In this subanalysis of severe patients, the success rate at week 16 was higher in the
IVM-treated group than in the MTZ-treated group $(82.5 \%$ vs. $63 \%$ IGA 0 or $1 ; P=0.005)$. The superiority of IVM $1 \%$ was more pronounced in this subpopulation of severe subjects (between-group difference of 19.5\%) than in the overall population of moderate-to-severe subjects, in which the success rate was reported to be $84.9 \%$ IVM $1 \%$ vs. $75.4 \%$ MTZ $0.75 \%$ at week $16(P<0.001$; between-group difference of 9.5\%) [8].

Archive photographs are shown in Fig. 5 to illustrate a severe subject (IGA of 4 at baseline) who had an IGA of 1 after 14 weeks treatment
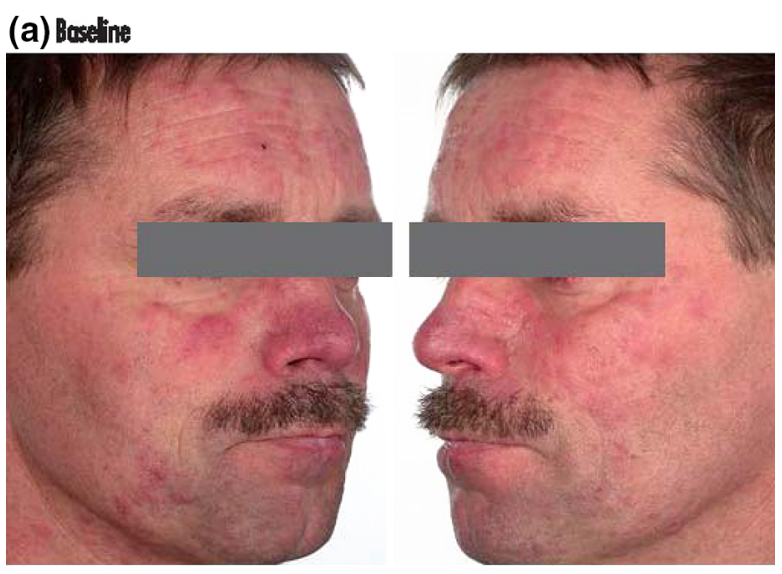

(b) Woek 14

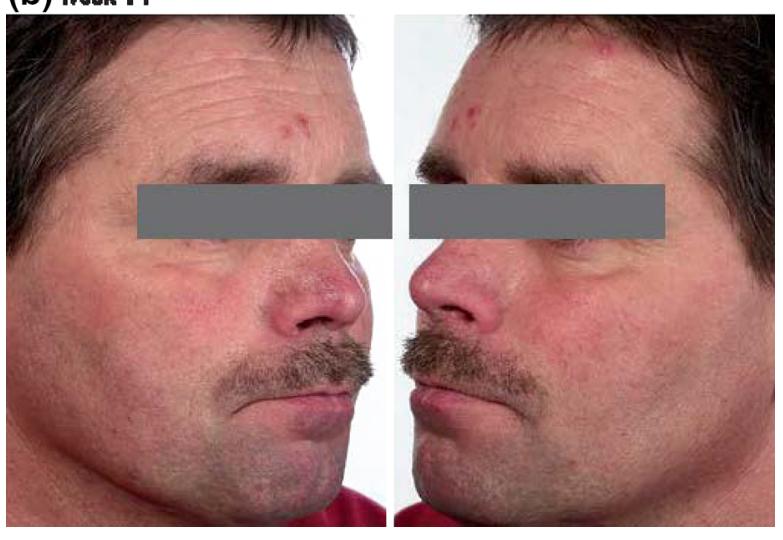

Fig. 5 Photographs of a severe subject with a an IGA of 4 before treatment and $\mathbf{b}$ an IGA of 1 after 14 weeks treatment with ivermectin $1 \%$ cream once daily. $I G A$ investigator's global assessment. Informed consent was received from the patient for publication of these photographs 
with IVM $1 \%$ cream QD. As the IGA is a composite score including both inflammatory lesions and erythema, it is difficult to obtain a score of 0 (clear) since a combination treatment may be required. Of the successfully treated subjects (IGA score of 0 or 1 ), the proportion of severe subjects with complete healing of both inflammatory lesions and erythema lesions (an IGA score of 0 ) at week 16 was $27.5 \%$ in the IVM $1 \%$ group compared to $12.3 \%$ in the MTZ $0.75 \%$ group. The difference between an IGA score of 0 and 1 is clinically relevant to the patients since they want a full reduction of lesions and erythema.

The significantly greater reduction in lesion count at week 16 in the IVM 1\% group ( $-85.1 \%$ vs. $-75.2 \%$ for $\mathrm{MTZ} 0.75 \% ; \quad P=0.011$ ) translated into a clinically meaningful difference for the investigator (reduction in IGA score) and for the subject since more subjects in the IVM $1 \%$ group (85.9\%) rated the improvement in their rosacea as good or excellent at week 16 compared to the MTZ $0.75 \%$ group $(67.5 \%)$.

At week 52, the overall success rate of subjects who never relapsed was higher in the IVM $1 \%$ group than the MTZ $0.75 \%$ group (23.2\% IVM $1 \%$ vs. $12.3 \%$ MTZ $0.75 \%$ ), which is important from a pharmacoeconomic perspective, in addition to the fact that IVM $1 \%$ is QD and so requires a lower quantity of product per treatment compared to MTZ 0.75\% (BID).

Comparable safety results demonstrated that both products were very well tolerated, which is important as rosacea patients tend to have highly sensitive skin. The results in this group of severe subjects are consistent with a previous long-term safety study on IVM $1 \%$ cream in moderate-to-severe subjects [15].

Severe inflammatory lesions of rosacea are often treated with oral antibiotics, particularly tetracyclines, despite a poor safety profile $[6,16]$. However, antibiotic resistance is now a major threat to public health causing people to be sick for longer and increasing the cost of health care with lengthier stays in hospital [5]. Dermatologists contribute to overall antibiotic use and tend to prescribe antibiotics chronically, particularly for acne and rosacea [17]. Hence, there is a need to find effective alternatives to antibiotics or sub-anti-microbial dosage forms. One such example is the use of a sub-anti-microbial dosage of doxycycline (40 mg modified-release doxycycline), which has anti-inflammatory rather than anti-microbial effect, that has been reported to be as effective as $100 \mathrm{mg}$ with significantly fewer gastrointestinal side effects [18]. Since ivermectin is a member of the avermectin class of compounds (with anti-inflammatory and anti-parasitic effects) and is not an antibiotic, it is thus an effective alternative treatment for papulopustular rosacea, even in severe patients.

Limitations of this subanalysis include the small number of subjects. However, despite the small sample size, statistical significance was reached for the efficacy endpoints. Another limitation was the absence of double-blind due to the fact that IVM $1 \%$ is QD, whereas MTZ $0.75 \%$ is BID, as well as the obvious difference in appearance of the two creams. However, a QD treatment has the advantage of being more appealing to patients, especially when it is more efficacious.

\section{CONCLUSIONS}

In patients with severe papulopustular rosacea, better efficacy with IVM 1\% cream compared to MTZ $0.75 \%$ cream contributes to an improved QoL with significantly more patients achieving an MCID in DLQI score and higher mean EQ-5D 
score. IVM 1\% cream QD is thus a better alternative than MTZ $0.75 \%$ cream BID for severe papulopustular rosacea patients.

\section{ACKNOWLEDGMENTS}

Sponsorship and article processing charges for this study were funded by Galderma $R \& D$ (Sophia Antipolis, France). Medical writing and editorial assistance in the preparation of this manuscript were provided by Helen Simpson, PhD, of Galderma R \& D. All named authors meet the International Committee of Medical Journal Editors (ICMJE) criteria for authorship for this manuscript, take responsibility for the integrity of the work as a whole, and have given final approval for the version to be published.

Disclosures. Martin Schaller has been a member of Galderma and Marpinion advisory boards for the past two years; has received lecture fees from AbbVie, Bayer Healthcare, Galderma, and La Roche-Posay; and has received research grants from Galderma and Bayer Healthcare. Thomas Dirschka has received research grants from Galderma; has been a lecturer for Almirall, Galderma, Leo, Meda, Novartis; and a member of advisory boards of Almirall, Galderma, Glaxo Smithkline, Leo, Meda, Dr. Pfleger, Riemser, Janssen, Novartis. Lajos Kemény has received research grants from Galderma; has been a lecturer for Abbvie, Galderma, Janssen, Novartis, Sager; and a member of advisory boards of Abbvie, Janssen, Novartis. Philippe Briantais is an employee of Galderma R\&D. Jean Jacovella is an employee of Galderma R\&D.

Compliance with Ethics Guidelines. This article is based on a previously conducted study and does not involve any new studies of human or animal subjects performed by any of the authors. Informed consent was received from the patient for publication of the photographs.

Open Access. This article is distributed under the terms of the Creative Commons Attribution-NonCommercial 4.0 International License (http://creativecommons.org/licenses/ by-nc/4.0/), which permits any noncommercial use, distribution, and reproduction in any medium, provided you give appropriate credit to the original author(s) and the source, provide a link to the Creative Commons license, and indicate if changes were made.

\section{REFERENCES}

1. Tan J, Schöfer H, Araviiskaia E, Audibert F, Kerrouche N, Berg M, RISE study group. Prevalence of rosacea in the general population of Germany and Russia-the RISE study. J Eur Acad Dermatol Venereol. 2016;30(3):428-34.

2. Wilkin J, Dahl M, Detmar M, et al. National Rosacea Society Expert Committee. Standard grading system for rosacea: report of the National Rosacea Society Expert Committee on the classification and staging of rosacea. J Am Acad Dermatol. 2004;50(6):907-12.

3. Aksoy B, Altaykan-Hapa A, Egemen D, Karagöz F, Atakan N. The impact of rosacea on quality of life: effects of demographic and clinical characteristics and various treatment modalities. $\mathrm{Br} \mathrm{J}$ Dermatol. 2010;163(4):719-25.

4. European Commission. Antimicrobial resistanceaction at EU Level. http://ec.europa.eu/dgs/health_ food-safety/amr/action_eu/index_en.htm. Accessed 12 Apr 2016.

5. World Health Organization. Global action plan on antimicrobial resistance. http://www.who.int/ drugresistance/global_action_plan/en/. Accessed 12 Apr 2016.

6. van Zuuren EJ, Fedorowicz Z, Carter B, van der Linden MMD, Charland L. Interventions for rosacea. Cochrane Database Syst Rev. 2015;4:CD003262.

7. Stein L, Kircik L, Fowler J, et al. Efficacy and safety of ivermectin $1 \%$ cream in treatment of 
papulopustular rosacea: results of two randomized, double-blind, vehicle-controlled pivotal studies. J Drugs Dermatol. 2014;13(3):316-23.

8. Taieb A, Ortonne JP, Ruzicka T, et al. Ivermectin Phase III study group. Superiority of ivermectin 1\% cream over metronidazole $0.75 \%$ cream in treating inflammatory lesions of rosacea: a randomized, investigator-blinded trial. $\mathrm{Br} \mathrm{J}$ Dermatol. 2015;172(4):1103-10.

9. Taieb A, Khemis A, Ruzicka T, et al. Ivermectin Phase III Study Group. Maintenance of remission following successful treatment of papulopustular rosacea with ivermectin $1 \%$ cream vs. metronidazole $0.75 \%$ cream: 36 -week extension of the ATTRACT randomized study. J Eur Acad Dermatol Venereol. 2016;30(5):829-36.

10. EuroQol Group. EuroQol—a new facility for the measurement of health-related quality of life. Health Policy. 1990;16:199-208.

11. Finlay AY, Khan GK. Dermatology Life Quality Index (DLQI) - a simple practical measure for routine clinical use. Clin Exp Dermatol. 1994;19:210-6.

12. Lamers LM, Stalmeier PF, McDonnell J, Krabbe PF, van Busschbach JJ. Measuring the quality of life in economic evaluations: the Dutch EQ-5D tariff]. [Article in Dutch. Ned Tijdschr Geneeskd. 2005;149(28):1574-8.
13. Walters SJ, Brazier JE. Comparison of the minimally important difference for two health state utility measures: EQ-5D and SF-6D. Qual Life Res. 2005;14(6):1523-32.

14. Basra MK, Salek MS, Camilleri L, Sturkey R, Finlay AY. Determining the minimal clinically important difference and responsiveness of the Dermatology Life Quality Index (DLQI): further data. Dermatology. 2015;230(1):27-33.

15. Stein Gold L, Kircik L, Fowler J, et al. Ivermectin Phase 3 Study Group. Long-term safety of ivermectin $1 \%$ cream vs azelaic acid $15 \%$ gel in treating inflammatory lesions of rosacea: results of two 40-week controlled, investigator-blinded trials. J Drugs Dermatol. 2014;13(11):1380-6.

16. Li WQ, Cho E, Khalili H, Wu S, Chan AT, Qureshi AA. Rosacea, use of tetracycline, and risk of incident inflammatory bowel disease in women. Clin Gastroenterol Hepatol. 2016;14(2):220-5.

17. Walsh TR, Efthimiou J, Dréno B. Systematic review of antibiotic resistance in acne: an increasing topical and oral threat. Lancet Infect Dis. 2016;16(3):e23-33.

18. Del Rosso JQ, Schlessinger J, Werschler P. Comparison of anti-inflammatory dose doxycycline versus doxycycline $100 \mathrm{mg}$ in the treatment of rosacea. J Drugs Dermatol. 2008;7(6):573-6. 